\title{
USAGE OF THE HOMOTOPY ANALYSIS METHOD FOR SOLVING FRACTIONAL VOLTERRA-FREDHOLM INTEGRO-DIFFERENTIAL EQUATION OF THE SECOND KIND
}

\author{
AHMED A. HAMOUD AND KIRTIWANT P. GHADLE
}

\begin{abstract}
The reliability of the homotopy analysis method (HAM) and reduction in the size of the computational work give this method a wider applicability. In this paper, HAM has been successfully applied to find the approximate solutions of Caputo fractional Volterra-Fredholm integro-differential equations. Also, the behavior of the solution can be formally determined by analytical approximation. Moreover, the study proves the existence and uniqueness results and the convergence of the solution. This paper concludes with an example to demonstrate the validity and applicability of the proposed technique.
\end{abstract}

\section{Introduction}

The integro-differential equations of fractional order have attracted more attention of physicists and mathematicians which provide an efficiency for the description of many practical dynamical arising in engineering and scientific disciplines such as, physics, biology, electrochemistry, electromagnetic, control theory and viscoelasticity $[2,3$, 4, 5, 6, 13, 15, 17]. Recently, many authors focus on the development of numerical and analytical techniques for fractional integro-differential equations. Al-Samadi and Gumah [1] applied the HAM for solving fractional SEIR epidemic model, Zurigat et al. [21] applied HAM for system of Caputo fractional integro-differential equations. Yang and Hou [17] applied the Laplace decomposition method to solve the Caputo fractional integro-differential equations, Mittal and Nigam [15] applied the Adomian decomposition method to approximate solutions for Caputo fractional integro-differential equations, and Ma and Huang [13] applied hybrid collocation method to study integro-differential equations of fractional order. Moreover, properties of the fractional integro-differential equations have been studied by several authors $[1,18,19,21]$. The HAM,

Received January 17, 2018, accepted April 27, 2018.

2010 Mathematics Subject Classification. 26A33, 35C10, 65H20.

Key words and phrases. Homotopy analysis method, Caputo fractional Volterra-Fredholm integrodifferential equation, existence and uniqueness results.

Corresponding author: Ahmed A. Hamoud. 
that was first proposed by Liao [11, 12], is implemented to derive analytic approximate solutions of Caputo fractional integro-differential equations and convergence of HAM for this kind of equations is considered. Unlike all other analytical methods, HAM adjusts and controls the convergence region of the series solution via an auxiliary parameter $\hbar$.

In this paper, we consider Caputo fractional Volterra-Fredholm integro-differential equations of the form:

$$
{ }^{c} D^{\alpha} u(x)=a(x) u(x)+g(x)+\int_{0}^{x} K_{1}(x, t) F_{1}(u(t)) d t+\int_{0}^{1} K_{2}(x, t) F_{2}(u(t)) d t,
$$

with the initial condition

$$
u(0)=u_{0}
$$

where ${ }^{c} D^{\alpha}$ is the Caputo's fractional derivative, $0<\alpha \leq 1, u: J \longrightarrow \mathbb{R}$, where $J=[0,1]$ be the continuous function which has to be determined, $g: J \longrightarrow \mathbb{R}$ and $K_{i}: J \times J \longrightarrow \mathbb{R}$, are continuous functions. $F_{i}: \mathbb{R} \longrightarrow \mathbb{R}, i=1,2$ are Lipschitz continuous functions.

The significance of this paper lies in its contribution as it:

1. Studies the behavior of the solution that can be formally determined by the analytical approximated method as the HAM.

2. Proves the existence, uniqueness of result us, and convergence of the solution of the Caputo fractional Volterra-Fredholm integro-differential equation.

The rest of the paper is organized as follows: In Section 2, some preliminaries and basic definitions related to fractional calculus are recalled. In Section 3, HAM is constructed for solving Caputo fractional Volterra-Fredholm integro-differential equations. In Section 4, the existence and uniqueness results and convergence of the solution are proved. In Section 5, the analytical example is presented to illustrate the accuracy of this method. Finally, the paper concludes with Section 6 which is the report of the study.

\section{Preliminaries}

Before we derive the HAM of the fractional integration, we first review some basic definitions of fractional calculus, which have been given in $[8,10,14,16,20]$.

Definition 1. The Riemann-Liouville fractional integral of order $\alpha>0$ of a function $f$ is defined as

$$
\begin{aligned}
J^{\alpha} f(x) & =\frac{1}{\Gamma(\alpha)} \int_{0}^{x}(x-t)^{\alpha-1} f(t) d t, \quad x>0, \quad \alpha \in \mathbb{R}^{+}, \\
J^{0} f(x) & =f(x),
\end{aligned}
$$

where $\mathbb{R}^{+}$is the set of positive real numbers. 
Definition 2. The fractional derivative of $f(x)$ in the Caputo sense is defined by

$$
\begin{aligned}
{ }^{c} D^{\alpha} f(x) & =J^{n-\alpha} D^{n} f(x) \\
& = \begin{cases}\frac{1}{\Gamma(n-\alpha)} \int_{0}^{x}(x-t)^{n-\alpha-1} \frac{d^{n} f(t)}{d t^{n}} d t, & n-1<\alpha<n, \\
\frac{d^{n} f(x)}{d x^{n}}, & \alpha=n,\end{cases}
\end{aligned}
$$

where the parameter $\alpha$ is the order of the derivative, in general it is real or even complex. But in this paper, we will consider $\alpha$ as positive real.

Hence, we have the following properties:

1. $J^{\alpha} J^{v} f=J^{\alpha+v} f, \quad \alpha, v>0$.

2. $J^{\alpha} x^{\beta}=\frac{\Gamma(\beta+1)}{\Gamma(\alpha+\beta+1)} x^{\alpha+\beta}, \quad \alpha>0, \beta>-1, \quad x>0$.

3. $J^{\alpha} D^{\alpha} f(x)=f(x)-\sum_{k=0}^{m-1} f^{(k)}\left(0^{+}\right) \frac{x^{k}}{k !}, \quad x>0, \quad m-1<\alpha \leq m$.

Definition 3. The Riemann-Liouville fractional derivative of order $\alpha>0$ is normally defined as

$$
D^{\alpha} f(x)=D^{m} J^{m-\alpha} f(x), \quad m-1<\alpha \leq m .
$$

Theorem 1 ([20] (Banach contraction principle)). Let $(X, d)$ be a complete metric space, then each contraction mapping $\mathscr{T}: X \longrightarrow X$ has a unique fixed point $x$ of $\mathscr{T}$ in $X$ i.e. $\mathscr{T} x=x$.

Theorem 2. ([9]) (Schauder's fixed point theorem). Let $X$ be a Banach space and let A a convex, closed subset of $X$. If $T: A \longrightarrow A$ be the map such that the set $\{T u: u \in A\}$ is relatively compact in $X$ (or $T$ is continuous and completely continouous). Then $T$ has at least one fixed point $u^{*} \in A: T u^{*}=u^{*}$.

\section{Homotopy Analysis Method (HAM)}

In order to show the basic idea of HAM, consider the following differential equation [7]:

$$
N[u]=0,
$$

where $N$ is a nonlinear operator, $u(x)$ is unknown function and $x$ is an independent variable. We can construct a homotopy when considering, $N[u]=0$, as follows:

$$
(1-q) L\left[\phi(x ; q)-u_{0}(x)\right]-q \hbar H_{1}(x) N[\phi(x ; q)]=\hat{H}\left[\phi(x ; q) ; u_{0}(x), H_{1}(x), \hbar, q\right],
$$

where $u_{0}(x)$ denotes an initial guess of the exact solution $u(x), \hbar \neq 0$ an auxiliary parameter, $H_{1}(x) \neq 0$ an auxiliary function, and $L$ an auxiliary linear operator with the property $L[s(x)]=0$ 
when $s(x)=0$, and using $q \in[0,1]$ as an embedding parameter. It should be asserted that we have great liberty to select the initial guess $u_{0}(x)$, the auxiliary linear operator $L$, the non-zero auxiliary parameter $\hbar$, and the auxiliary function $H_{1}(x)$. Enforcing the homotopy Eq.(6) to be zero, i.e.,

$$
H_{1}\left[\phi(x ; q) ; u_{0}(x), H_{1}(x), \hbar, q\right]=0,
$$

we have the so-called zero-order deformation equation

$$
(1-q) L\left[\phi(x ; q)-u_{0}(x)\right]=q \hbar H_{1}(x) N[\phi(x ; q)]
$$

when $q=0$, the zero-order deformation Eq.(8) becomes

$$
\phi(x ; 0)=u_{0}(x)
$$

and when $q=1$, since $\hbar \neq 0$ and $H_{1}(x) \neq 0$, the zero-order deformation Eq.(8) is equivalent to

$$
\phi(x ; 1)=u(x) .
$$

Thus, according to Eqs.(9) and (10), as the embedding parameter $q$ increases from 0 to $1, \phi(x ; q)$ varies continuously from the initial approximation $u_{0}(x)$ to the exact solution $u(x)$. Such a kind of continuous variation is called deformation in homotopy [11,21]. Due to Taylor's theorem, $\phi(x ; q)$ can be expanded in power series of $q$ as follows

$$
\phi(x ; q)=u_{0}(x)+\sum_{m=1}^{\infty} u_{m}(x) q^{m}
$$

where,

$$
u_{m}(x)=\left.\frac{1}{m !} \frac{\partial^{m} \phi(x ; q)}{\partial q^{m}}\right|_{q=0} .
$$

Let the initial guess $u_{0}(x)$, the auxiliary linear parameter $L$, the nonzero auxiliary parameter $\hbar$ and the auxiliary function $H_{1}(x)$ be properly chosen so that the power series (11) of $\phi(x ; q)$ converges at $q=1$, then, we have under these assumptions the solution series

$$
u(x)=\phi(x ; 1)=u_{0}(x)+\sum_{m=1}^{\infty} u_{m}(x)
$$

From Eq.(11), we can write Eq.(8) as follows:

$$
\begin{aligned}
(1-q) L\left[\phi(x ; q)-u_{0}(x)\right] & =(1-q) L\left[\sum_{m=1}^{\infty} u_{m}(x) q^{m}\right] \\
& =q \hbar H_{1}(x) N[\phi(x ; q)],
\end{aligned}
$$

then,

$$
L\left[\sum_{m=1}^{\infty} u_{m}(x) q^{m}\right]-q L\left[\sum_{m=1}^{\infty} u_{m}(x) q^{m}\right]=q \hbar H_{1}(x) N[\phi(x ; q)]
$$


By differentiating Eq.(15) $m$-times with respect to $q$ and evaluating at $q=0$, we obtain

$$
\begin{aligned}
\left\{L\left[\sum_{m=1}^{\infty} u_{m}(x) q^{m}\right]-q L\left[\sum_{m=1}^{\infty} u_{m}(x) q^{m}\right]\right\}^{(m)} & =q \hbar H_{1}(x) N[\phi(x ; q)]^{(m)} \\
& =m ! L\left[u_{m}(x)-u_{m-1}(x)\right] \\
& =\left.\hbar H_{1}(x) m \frac{\partial^{m-1} N[\phi(x ; q)]}{\partial q^{m-1}}\right|_{q=0} .
\end{aligned}
$$

Therefore,

$$
L\left[u_{m}(x)-\chi_{m} u_{m-1}(x)\right]=\hbar H_{1}(x) \Re_{m}\left(\overrightarrow{u_{m-1}}(x)\right),
$$

where,

$$
\Re_{m}\left(\overrightarrow{u_{m-1}}(x)\right)=\left.\frac{1}{(m-1) !} \frac{\partial^{m-1} N[\varphi(x ; q)]}{\partial q^{m-1}}\right|_{q=0},
$$

and

$$
\chi_{m}= \begin{cases}0 & m \leq 1 \\ 1 & m>1\end{cases}
$$

Note that the high-order deformation Eq.(16) is governing the linear operator $L$, and the term $\Re_{m}\left(\overrightarrow{u_{m-1}}(x)\right)$ can be expressed simply by Eq.(17) for any nonlinear operator $N$.

\section{HAM applied to fractional Volterra-Fredholm integro-differential equation:}

We consider Caputo fractional Volterra-Fredholm integro-differential equations given by (1)-(2), we can define

$$
\begin{aligned}
N[\phi(x ; q)]= & { }^{c} D^{\alpha} \phi(x ; q)-a(x) \phi(x ; q)-g(x)-\int_{0}^{x} K_{1}(x, t) F_{1}(\phi(t ; q)) d t \\
& -\int_{0}^{1} K_{2}(x, t) F_{2}(\phi(t ; q)) d t .
\end{aligned}
$$

Now we construct the zero-order deformation equation

$$
(1-q)^{c} D^{\alpha}\left[\phi(x ; q)-u_{0}(x)\right]=q \hbar N[\phi(x ; q)]
$$

subject to the following initial conditions

$$
u_{0}(x)=\phi(0 ; q)=u_{0}
$$

where $q \in[0,1]$ is the embedding parameter, $\hbar \neq 0$ is an auxiliary parameter, $u_{0}(x)$ is an initial guess of the solution $u(x)$ and $\phi(x ; q)$ is an unknown function on the independent variables $x$ and $q$. Also we suppose that

$$
{ }^{c} D^{\alpha}(C)=0,
$$


where $C$ is an integral constant. When the parameter $q$ increases from 0 to 1 , then the homotopy solution $\phi(x ; q)$ varies from $u_{0}(x)$ to solution $u(x)$ of the original equation (1). Using the parameter $q, \phi(x ; q)$ can be expanded in Taylor series as follows:

$$
\phi(x ; q)=u_{0}(x)+\sum_{m=1}^{\infty} u_{m}(x) q^{m},
$$

where $u_{m}(x)$ defines as (12).

Assuming that the auxiliary parameter $\hbar$ is properly selected so that the above series is convergent when $q=1$, then the solution $u(x)$ can be given by

$$
u(x)=u_{0}(x)+\sum_{m=1}^{\infty} u_{m}(x) .
$$

Differentiating (19) and the initial condition (20) $m$ times with respect to $q$, then setting $q=0$, and finally dividing them by $m$ !, we get the $m^{t h}$-order deformation equation

$$
{ }^{c} D^{\alpha}\left[u_{m}(x)-\chi_{m} u_{m-1}(x)\right]=\hbar \Re_{m}\left(\overrightarrow{u_{m-1}}(x)\right),
$$

subject to the following initial conditions,

$$
u_{m}(0)=0
$$

where,

$$
\begin{aligned}
\Re_{m}\left(\overrightarrow{u_{m-1}}(x)\right)= & \left.\frac{1}{(m-1) !} \frac{\partial^{m-1} N[\phi(x ; q)]}{\partial q^{m-1}}\right|_{q=0}, \\
= & { }^{c} D^{\alpha} u_{m-1}(x)-a(x) u_{m-1}(x)-\int_{0}^{x} K_{1}(x, t) F_{1}\left(u_{m-1}(t)\right) d t \\
& -\int_{0}^{1} K_{2}(x, t) F_{2}\left(u_{m-1}(t)\right) d t-\left(1-\chi_{m}\right) g(x)
\end{aligned}
$$

and

$$
\overrightarrow{u_{m}}=u_{0}, u_{1}, \cdots, u_{m}
$$

Applying the operator $J^{\alpha}$ to both sides of the linear $m$-order deformation (24)

$$
\begin{aligned}
u_{m}(x)= & \left(\chi_{m}+\hbar\right) u_{m-1}(x)-\hbar J^{\alpha}\left[a(x) u_{m-1}(x)\right. \\
& \left.+\int_{0}^{x} K_{1}(x, t) F_{1}\left(u_{m-1}(t)\right) d t+\int_{0}^{1} K_{2}(x, t) F_{2}\left(u_{m-1}(t)\right) d t+\left(1-\chi_{m}\right) g(x)\right] .
\end{aligned}
$$

\section{The main results}

In this section, we shall give an existence and uniqueness results of (1) - (2) and prove it.

Before starting and proving the main results, we introduce the following hypotheses: 
(A1) There exists two constants $L_{F_{1}}, L_{F_{2}}>0$ such that, for any $u_{1}, u_{2} \in C(J, \mathbb{R})$

$$
\left|F_{1}\left(u_{1}(x)\right)-F_{1}\left(u_{2}(x)\right)\right| \leq L_{F_{1}}\left|u_{1}-u_{2}\right|
$$

and

$$
\left|F_{2}\left(u_{1}(x)\right)-F_{2}\left(u_{2}(x)\right)\right| \leq L_{F_{2}}\left|u_{1}-u_{2}\right|
$$

(A2) There exists two functions $K_{1}^{*}, K_{2}^{*} \in C\left(D, \mathbb{R}^{+}\right)$, the set of all positive function continuous on $D=\{(x, t) \in \mathbb{R} \times \mathbb{R}: 0 \leq t \leq x \leq 1\}$ such that $K_{1}^{*}=\sup _{x \in[0,1]} \int_{0}^{x}\left|K_{1}(x, t)\right| d t<\infty, K_{2}^{*}=$ $\sup _{x \in[0,1]} \int_{0}^{x}\left|K_{2}(x, t)\right| d t<\infty$.

(A3) The two functions $a, g: J \rightarrow \mathbb{R}$ are continuous.

Lemma 1. Let $u_{0}(x) \in C(J, \mathbb{R})$, then $u(t) \in C\left(J, \mathbb{R}^{+}\right)$is a solution of the problem (1) - (2) iff $u$ satisfies

$$
\begin{aligned}
u(x)= & u_{0}+\frac{1}{\Gamma(\alpha)} \int_{0}^{x}(x-s)^{\alpha-1} a(s) u(s) d s+\frac{1}{\Gamma(\alpha)} \int_{0}^{x}(x-s)^{\alpha-1} g(s) d s \\
& +\frac{1}{\Gamma(\alpha)} \int_{0}^{x}(x-s)^{\alpha-1}\left(\int_{0}^{s} K_{1}(s, \tau) F_{1}(u(\tau)) d \tau+\int_{0}^{1} K_{2}(s, \tau) F_{2}(u(\tau)) d \tau\right) d s,
\end{aligned}
$$

for $x \in J$.

Our first result is based on the Banach contraction principle.

Theorem 3. Assumes that (A1), (A2) and (A3) hold. If

$$
\left(\frac{\|a\|_{\infty}+K_{1}^{*} L_{F_{1}}+K_{2}^{*} L_{F_{2}}}{\Gamma(\alpha+1)}\right)<1 .
$$

Then there exists a unique solution $u(t) \in C(J)$ to $(1)-(2)$.

Proof. By Lemma 1. we know that a function $u$ is a solution to (1) - (2) iff $u$ satisfies

$$
\begin{aligned}
u(x) & =u_{0}+\frac{1}{\Gamma(\alpha)} \int_{0}^{x}(x-s)^{\alpha-1} a(s) u(s) d s+\frac{1}{\Gamma(\alpha)} \int_{0}^{x}(x-s)^{\alpha-1} g(s) d s \\
& +\frac{1}{\Gamma(\alpha)} \int_{0}^{x}(x-s)^{\alpha-1}\left(\int_{0}^{s} K_{1}(s, \tau) F_{1}(u(\tau)) d \tau+\int_{0}^{1} K_{2}(s, \tau) F_{2}(u(\tau)) d \tau\right) d s
\end{aligned}
$$

Let the operator $T: C(J, \mathbb{R}) \rightarrow C(J, \mathbb{R})$ be defined by

$$
\begin{aligned}
(T u)(x)= & u_{0}+\frac{1}{\Gamma(\alpha)} \int_{0}^{x}(x-s)^{\alpha-1} a(s) u(s) d s+\frac{1}{\Gamma(\alpha)} \int_{0}^{x}(x-s)^{\alpha-1} g(s) d s \\
& +\frac{1}{\Gamma(\alpha)} \int_{0}^{x}(x-s)^{\alpha-1}\left(\int_{0}^{s} K_{1}(\tau, x) F_{1}(u(\tau)) d \tau+\int_{0}^{1} K_{2}(s, \tau) F_{2}(u(\tau)) d \tau\right) d s
\end{aligned}
$$


we can see that, if $u \in C(J, \mathbb{R})$ is a fixed point of $T$, then $u$ is a solution of (1), (2).

Now we prove $T$ has a fixed point $u$ in $C(J, \mathbb{R})$. For that, let $u_{1}, u_{2} \in C(J, \mathbb{R})$ and for any $x \in[0,1]$ such that

$$
\begin{aligned}
u_{1}(x)= & u_{0}+\frac{1}{\Gamma(\alpha)} \int_{0}^{x}(x-s)^{\alpha-1} a(s) u_{1}(s) d s+\frac{1}{\Gamma(\alpha)} \int_{0}^{x}(x-s)^{\alpha-1} g(s) d s \\
& +\frac{1}{\Gamma(\alpha)} \int_{0}^{x}(x-s)^{\alpha-1}\left(\int_{0}^{s} K_{1}(s, \tau) F_{1}\left(u_{1}(\tau)\right) d \tau+\int_{0}^{1} K_{2}(s, \tau) F_{2}\left(u_{1}(\tau)\right) d \tau\right) d s,
\end{aligned}
$$

and,

$$
\begin{aligned}
u_{2}(x)= & u_{0}+\frac{1}{\Gamma(\alpha)} \int_{0}^{x}(x-s)^{\alpha-1} a(s) u_{2}(s) d s+\frac{1}{\Gamma(\alpha)} \int_{0}^{x}(x-s)^{\alpha-1} g(s) d s \\
& +\frac{1}{\Gamma(\alpha)} \int_{0}^{x}(x-s)^{\alpha-1}\left(\int_{0}^{s} K_{1}(s, \tau) F_{1}\left(u_{2}(\tau)\right) d \tau+\int_{0}^{1} K_{2}(s, \tau) F_{2}\left(u_{2}(\tau)\right) d \tau\right) d s .
\end{aligned}
$$

Consequently, we get

$$
\begin{aligned}
& \left|\left(T u_{1}\right)(x)-\left(T u_{2}\right)(x)\right| \\
& \leq \frac{1}{\Gamma(\alpha)} \int_{0}^{x}(x-s)^{\alpha-1}|a(s)|\left|u_{1}(s)-u_{2}(s)\right| d s \\
& \quad+\frac{1}{\Gamma(\alpha)} \int_{0}^{x}(x-s)^{\alpha-1}\left(\begin{array}{c}
\int_{0}^{s}\left|K_{1}(s, \tau)\right|\left|F_{1}\left(u_{1}(\tau)\right)-F_{1}\left(u_{2}(\tau)\right)\right| d \tau \\
+\int_{0}^{1}\left|K_{2}(s, \tau)\right|\left|F_{2}\left(u_{1}(\tau)\right)-F_{2}\left(u_{2}(\tau)\right)\right| d \tau
\end{array}\right) d s \\
& \leq \frac{\|a\|_{\infty}}{\Gamma(\alpha+1)}\left|u_{1}(x)-u_{2}(x)\right|+\frac{K_{1}^{*} L_{F_{1}}}{\Gamma(\alpha+1)}\left|u_{1}(x)-u_{2}(x)\right|+\frac{K_{2}^{*} L_{F_{2}}}{\Gamma(\alpha+1)}\left|u_{1}(x)-u_{2}(x)\right| \\
& =\left(\frac{\|a\|_{\infty}+K_{1}^{*} L_{F_{1}}+K_{2}^{*} L_{F_{2}}}{\Gamma(\alpha+1)}\right)\left|u_{1}(x)-u_{2}(x)\right|,
\end{aligned}
$$

From the inequality (28) we have

$$
\left\|T u_{1}-T u_{2}\right\|_{\infty}<\left\|u_{1}-u_{2}\right\|_{\infty}
$$

This means that $T$ is a contraction map. By the Banach contraction principle, we can conclude that $T$ has a unique fixed point $u$ in $C(J, \mathbb{R})$.

Now, we will study the existence result by means of Schauder's fixed point theorem.

Theorem 4. Assumes that $F_{1}, F_{2}$ are continuous functions and (A2), (A3) hold, if

$$
\frac{\|a\|_{\infty}}{\Gamma(\alpha+1)}<1
$$

Then there exists at least a solution $u(x) \in C(J, \mathbb{R})$ to problem $(1)-(2)$.

Proof. Let the operator $T: C(J, \mathbb{R}) \rightarrow C(J, \mathbb{R})$, be defined as in Theorem 3, firstly, we prove that the operator $T$ is completely continuous. 
(1) We show that $T$ is continuous.

Let $u_{n}$ be a sequence such that $u_{n} \rightarrow u$ in $C(J, \mathbb{R})$. Then for each $u_{n}, u \in C(J, \mathbb{R})$ and for any $x \in J$ we have

$$
\begin{aligned}
& \left|\left(T u_{n}\right)(x)-(T u)(x)\right| \leq \frac{1}{\Gamma(\alpha)} \int_{0}^{x}(x-s)^{\alpha-1}|a(s)|\left|u_{n}(s)-u(s)\right| d s \\
& +\frac{1}{\Gamma(\alpha)} \int_{0}^{x}(x-s)^{\alpha-1}\left(\begin{array}{c}
\int_{0}^{s}\left|K_{1}(s, \tau)\right|\left|F_{1}\left(u_{n}(\tau)\right)-F_{1}(u(\tau))\right| d \tau \\
+\int_{0}^{1}\left|K_{2}(s, \tau)\right|\left|F_{2}\left(u_{n}(\tau)\right)-F_{2}(u(\tau))\right| d \tau
\end{array}\right) d s \\
& \leq \frac{1}{\Gamma(\alpha)} \int_{0}^{x}(x-s)^{\alpha-1} \sup _{s \in J}|a(s)| \sup _{s \in J}\left|u_{n}(s)-u(s)\right| d s \\
& +\frac{1}{\Gamma(\alpha)} \int_{0}^{x}(x-s)^{\alpha-1}\left(\begin{array}{c}
\sup _{s, \tau \in J} \int_{0}^{\tau}\left|K_{1}(s, \tau)\right| \sup _{\tau \in J}\left|F_{1}\left(u_{n}(\tau)\right)-F_{1}(u(\tau))\right| d \tau \\
+\sup _{s, \tau \in J} \int_{0}^{1}\left|K_{2}(s, \tau)\right| \sup _{\tau \in J}\left|F_{2}\left(u_{n}(\tau)\right)-F_{2}(u(\tau))\right| d \tau
\end{array}\right) d s \\
& \leq\|a\|_{\infty}\left\|u_{n}(.)-u(.)\right\|_{\infty} \frac{1}{\Gamma(\alpha)} \int_{0}^{x}(x-s)^{\alpha-1} d s \\
& +K_{1}^{*}\left\|F_{1}\left(u_{n}(.)\right)-F_{1}(u(.))\right\|_{\infty} \frac{1}{\Gamma(\alpha)} \int_{0}^{x}(x-s)^{\alpha-1} d s \\
& +K_{2}^{*}\left\|F_{2}\left(u_{n}(.)\right)-F_{2}(u(.))\right\|_{\infty} \frac{1}{\Gamma(\alpha)} \int_{0}^{x}(x-s)^{\alpha-1} d s .
\end{aligned}
$$

Since $\int_{0}^{x}(x-s)^{\alpha-1} d s$ is bounded, $\lim _{n \rightarrow \infty} u_{n}(x)=u(x)$ and $F_{1}, F_{2}$ are continuous functions, we conclude that $\left\|T u_{n}-T u\right\|_{\infty} \rightarrow 0$ as $n \rightarrow \infty$, thus, $T$ is continuous on $C(J, \mathbb{R})$.

(2) We verify that $T$ maps bounded sets into bounded sets in $C(J, \mathbb{R})$.

Indeed, just we show that for any $\lambda>0$ there exists a positive constant $\ell$ such that for each $u \in \mathbb{B}_{\lambda}=\left\{u \in C(J, \mathbb{R}):\|u\|_{\infty} \leq \lambda\right\}$, one has $\|T u\|_{\infty} \leq \ell$.

Let $\mu_{1}=\sup _{(u) \in J \times[0, \lambda]} F_{1}(u(x))+1$, and $\mu_{2}=\sup _{(u) \in J \times[0, \lambda]} F_{2}(u(x))+1$.

and for any $u \in \mathbb{B}_{r}$ and for each $x \in J$, we have

$$
\begin{aligned}
|(T u)(x)|= & \left|u_{0}\right|+\frac{1}{\Gamma(\alpha)} \int_{0}^{x}(x-s)^{\alpha-1}|a(s)||u(s)| d s+\frac{1}{\Gamma(\alpha)} \int_{0}^{x}(x-s)^{\alpha-1}|g(s)| d s \\
& +\frac{1}{\Gamma(\alpha)} \int_{0}^{x}(x-s)^{\alpha-1}\left(\int_{0}^{s}\left|K_{1}(s, \tau)\right|\left|F_{1}(u(\tau))\right| d \tau+\int_{0}^{1}\left|K_{2}(s, \tau)\right|\left|F_{2}(u(\tau))\right| d \tau\right) d s \\
\leq & \left|u_{0}\right|+\|u\|_{\infty}\|a\|_{\infty} \frac{x^{\alpha}}{\Gamma(\alpha+1)}+\|g\|_{\infty} \frac{x^{\alpha}}{\Gamma(\alpha+1)}+\frac{K_{1}^{*} \mu_{1} x^{\alpha}}{\Gamma(\alpha+1)}+\frac{K_{2}^{*} \mu_{2} x^{\alpha}}{\Gamma(\alpha+1)} \\
\leq & \left(\left|u_{0}\right|+\frac{\|a\|_{\infty} \lambda+\|g\|_{\infty}+K_{1}^{*} \mu_{1}+K_{2}^{*} \mu_{2}}{\Gamma(\alpha+1)}\right) \\
:= & \ell .
\end{aligned}
$$

Therefore, $\|T u\| \leq \ell$ for every $u \in \mathbb{B}_{r}$, which implies that $T \mathbb{B}_{r} \subset \mathbb{B}_{\ell}$. 
(3) We examine that $T$ maps bounded sets into equicontinuous sets of $C(J, \mathbb{R})$.

Let $\mathbb{B}_{\lambda}$ is defined as in (2) and for each $u \in \mathbb{B}_{\lambda}, x_{1}, x_{2} \in[0,1]$, with $x_{1}<x_{2}$ we have

$$
\begin{aligned}
& \left|(T u)\left(x_{2}\right)-(T u)\left(x_{1}\right)\right| \\
& \leq \frac{1}{\Gamma(\alpha)}\left|\int_{0}^{x_{2}}\left(x_{2}-s\right)^{\alpha-1} a(s) u(s) d s-\int_{0}^{x_{1}}\left(x_{1}-s\right)^{\alpha-1} a(s) u(s) d s\right| \\
& +\frac{1}{\Gamma(\alpha)}\left|\int_{0}^{x_{2}}\left(x_{2}-s\right)^{\alpha-1} g(s) d s-\int_{0}^{x_{1}}\left(x_{1}-s\right)^{\alpha-1} g(s) d s\right| \\
& +\frac{1}{\Gamma(\alpha)}\left|\begin{array}{l}
\int_{0}^{x_{2}}\left(x_{2}-s\right)^{\alpha-1}\left(\int_{0}^{s} K_{1}(s, \tau) F_{1}(u(\tau)) d \tau+\int_{0}^{1} K_{2}(s, \tau) F_{2}(u(\tau)) d \tau\right) d s \\
-\int_{0}^{x_{1}}\left(x_{1}-s\right)^{\alpha-1}\left(\int_{0}^{s} K_{1}(s, \tau) F_{1}(u(\tau)) d \tau+\int_{0}^{1} K_{2}(s, \tau) F_{2}(u(\tau)) d \tau\right) d s
\end{array}\right| \\
& \qquad \begin{array}{r}
\Gamma(\alpha)\left|\begin{array}{l}
\int_{0}^{x_{2}}\left(x_{2}-s\right)^{\alpha-1} a(s) u(s) d s-\int_{0}^{x_{1}}\left(x_{2}-s\right)^{\alpha-1} a(s) u(s) d s \\
+\int_{0}^{x_{1}}\left(x_{2}-s\right)^{\alpha-1} a(s) u(s) d s-\int_{0}^{x_{1}}\left(x_{1}-s\right)^{\alpha-1} a(s) u(s) d s
\end{array}\right| \\
+\frac{1}{\Gamma(\alpha)}\left|\begin{array}{l}
\int_{0}^{x_{2}}\left(x_{2}-s\right)^{\alpha-1} g(s) d s-\int_{0}^{x_{1}}\left(x_{2}-s\right)^{\alpha-1} g(s) d s \\
+\int_{0}^{x_{1}}\left(x_{2}-s\right)^{\alpha-1} g(s) d s-\int_{0}^{x_{1}}\left(x_{1}-s\right)^{\alpha-1} g(s) d s
\end{array}\right| \\
\quad \begin{array}{l}
\int_{0}^{x_{2}}\left(x_{2}-s\right)^{\alpha-1}\left(\int_{0}^{s} K_{1}(s, \tau) F_{1}(u(\tau)) d \tau+\int_{0}^{1} K_{2}(s, \tau) F_{2}(u(\tau)) d \tau\right) d s \\
-\int_{0}^{x_{1}}\left(x_{2}-s\right)^{\alpha-1}\left(\int_{0}^{s} K_{1}(s, \tau) F_{1}(u(\tau)) d \tau+\int_{0}^{1} K_{2}(s, \tau) F_{2}(u(\tau)) d \tau\right) d s \\
+\int_{0}^{x_{1}}\left(x_{2}-s\right)^{\alpha-1}\left(\int_{0}^{s} K_{1}(s, \tau) F_{1}(u(\tau)) d \tau+\int_{0}^{1} K_{2}(s, \tau) F_{2}(u(\tau)) d \tau\right) d s \\
-\int_{0}^{x_{1}}\left(x_{1}-s\right)^{\alpha-1}\left(\int_{0}^{s} K_{1}(s, \tau) F_{1}(u(\tau)) d \tau+\int_{0}^{1} K_{2}(s, \tau) F_{2}(u(\tau)) d \tau\right) d s .
\end{array} \mid
\end{array}
\end{aligned}
$$

Consequently,

$$
\begin{aligned}
& \left|(T u)\left(x_{2}\right)-(T u)\left(x_{1}\right)\right| \\
& \leq \frac{1}{\Gamma(\alpha)}\left(\begin{array}{c}
\int_{x_{1}}^{x_{2}}\left(x_{2}-s\right)^{\alpha-1}|a(s)||u(s)| d s \\
+\int_{0}^{x_{1}}\left(x_{1}-s\right)^{\alpha-1}-\left(x_{2}-s\right)^{\alpha-1}|a(s)||u(s)| d s
\end{array}\right) \\
& \quad+\frac{1}{\Gamma(\alpha)}\left(\begin{array}{c}
\int_{x_{1}}^{x_{2}}\left(x_{2}-s\right)^{\alpha-1}|g(s)| d s \\
+\int_{0}^{x_{1}}\left(x_{1}-s\right)^{\alpha-1}-\left(x_{2}-s\right)^{\alpha-1}|g(s)| d s
\end{array}\right) \\
& \quad+\frac{1}{\Gamma(\alpha)}\left(\begin{array}{c}
\int_{x_{1}}^{x_{2}}\left(x_{2}-s\right)^{\alpha-1}\left(\begin{array}{c}
\int_{0}^{s}\left|K_{1}(s, \tau)\right|\left|F_{1}(u(\tau))\right| d \tau \\
+\int_{0}^{1}\left|K_{2}(s, \tau)\right|\left|F_{2}(u(\tau))\right| d \tau
\end{array}\right) d s \\
+\int_{0}^{x_{1}}\left(x_{1}-s\right)^{\alpha-1}-\left(x_{2}-s\right)^{\alpha-1}\left(\begin{array}{c}
\int_{0}^{s}\left|K_{1}(s, \tau)\right|\left|F_{1}(u(\tau))\right| d \tau \\
+\int_{0}^{1}\left|K_{2}(s, \tau)\right|\left|F_{2}(u(\tau))\right| d \tau
\end{array}\right) d s
\end{array}\right) \\
& =I_{1}+I_{2}+I_{3},
\end{aligned}
$$

where

$$
\begin{aligned}
I_{1} & =\frac{1}{\Gamma(\alpha)}\left(\int_{x_{1}}^{x_{2}}\left(x_{2}-s\right)^{\alpha-1}|a(s)||u(s)| d s+\int_{0}^{x_{1}}\left(x_{1}-s\right)^{\alpha-1}-\left(x_{2}-s\right)^{\alpha-1}|a(s)||u(s)| d s\right) \\
& \leq \frac{\left(x_{2}-x_{1}\right)^{\alpha}}{\Gamma(\alpha+1)}\|a\|_{\infty} \lambda+\frac{x_{1}^{\alpha}}{\Gamma(\alpha+1)}\|a\|_{\infty} \lambda+\frac{\left(x_{2}-x_{1}\right)^{\alpha}}{\Gamma(\alpha+1)}\|a\|_{\infty} \lambda-\frac{x_{2}^{\alpha}}{\Gamma(\alpha+1)}\|a\|_{\infty} \lambda
\end{aligned}
$$




$$
\begin{aligned}
& =\frac{\|a\|_{\infty} \lambda}{\Gamma(\alpha+1)}\left(2\left(x_{2}-x_{1}\right)^{\alpha}+\left(x_{1}^{\alpha}-x_{2}^{\alpha}\right)\right) \\
& \leq \frac{\|a\|_{\infty} \lambda}{\Gamma(\alpha+1)} 2\left(x_{2}-x_{1}\right)^{\alpha}, \\
I_{2} & =\frac{1}{\Gamma(\alpha)}\left(\int_{x_{1}}^{x_{2}}\left(x_{2}-s\right)^{\alpha-1}|g(s)| d s+\int_{0}^{x_{1}}\left(x_{1}-s\right)^{\alpha-1}-\left(x_{2}-s\right)^{\alpha-1}|g(s)| d s\right) \\
& \leq \frac{\left(x_{2}-x_{1}\right)^{\alpha}}{\Gamma(\alpha+1)}\|g\|_{\infty}+\frac{x_{1}^{\alpha}}{\Gamma(\alpha+1)}\|g\|_{\infty}+\frac{\left(x_{2}-x_{1}\right)^{\alpha}}{\Gamma(\alpha+1)}\|g\|_{\infty}-\frac{x_{2}^{\alpha}}{\Gamma(\alpha+1)}\|g\|_{\infty} \\
& =\frac{\|g\|_{\infty}}{\Gamma(\alpha+1)}\left(2\left(x_{2}-x_{1}\right)^{\alpha}+\left(x_{1}^{\alpha}-x_{2}^{\alpha}\right)\right) \\
& \leq \frac{\|g\|_{\infty}}{\Gamma(\alpha+1)} 2\left(x_{2}-x_{1}\right)^{\alpha},
\end{aligned}
$$

and

$$
\begin{aligned}
& I_{3}= \frac{1}{\Gamma(\alpha)}+\int_{x_{1}}^{x_{2}}\left(x_{2}-s\right)^{\alpha-1}\left(\begin{array}{c}
\int_{0}^{s}\left|K_{1}(s, \tau)\right|\left|F_{1}(u(\tau))\right| d \tau \\
+\int_{0}^{1}\left|K_{2}(s, \tau)\right|\left|F_{2}(u(\tau))\right| d \tau
\end{array}\right) d s \\
&+\int_{0}^{x_{1}}\left(x_{1}-s\right)^{\alpha-1}-\left(x_{2}-s\right)^{\alpha-1}\left(\begin{array}{c}
\int_{0}^{s}\left|K_{1}(s, \tau)\right|\left|F_{1}(u(\tau))\right| d \tau \\
+\int_{0}^{1}\left|K_{2}(s, \tau)\right|\left|F_{2}(u(\tau))\right| d \tau
\end{array}\right) d s \\
& \leq \frac{\left(K_{1}^{*} \mu_{1}+K_{2}^{*} \mu_{2}\right)}{\Gamma(\alpha+1)}\left(2\left(x_{2}-x_{1}\right)^{\alpha}+\left(x_{1}^{\alpha}-x_{2}^{\alpha}\right)\right) \\
& \leq \frac{\left(K_{1}^{*} \mu_{1}+K_{2}^{*} \mu_{2}\right)}{\Gamma(\alpha+1)} 2\left(x_{2}-x_{1}\right)^{\alpha},
\end{aligned}
$$

we can conclude that the right-hand side of (30), (31) and (32) is independently of $u \in \mathbb{B}_{\lambda}$ and tends to zero as $x_{2}-x_{1} \rightarrow 0$. This leads to $\left|(T u)\left(x_{2}\right)-(T u)\left(x_{1}\right)\right| \rightarrow 0$ as $x_{2} \rightarrow x_{1}$. i.e. the set $\left\{T \mathbb{B}_{\lambda}\right\}$ is equicontinuous.

From $I_{1}$ to $I_{3}$ together with the Arzela-Ascoli theorem, we can conclude that $T: C(J, \mathbb{R}) \rightarrow$ $C(J, \mathbb{R})$ is completely continuous.

Finally, we need to investigate that there exists a closed convex bounded subset $\mathbb{B}_{\tilde{\lambda}}=$ $\left\{u \in C(J, \mathbb{R}):\|u\|_{\infty} \leq \widetilde{\lambda}\right\}$ such that $T \mathbb{B}_{\widetilde{\lambda}} \subseteq \mathbb{B}_{\tilde{\lambda}}$. For each positive integer $\tilde{\lambda}$, then $\mathbb{B}_{\widetilde{\lambda}}$ is clearly closed, convex and bounded of $C(J, \mathbb{R})$. We claim that there exists a positive integer $\tilde{\lambda}$ such that $T \mathbb{B}_{\epsilon} \subseteq \mathbb{B}_{\epsilon}$. If this property is false, then for every positive integer $\tilde{\lambda}$, there exists $u_{\tilde{\lambda}} \in \mathbb{B}_{\tilde{\lambda}}$ such that $\left(T u_{\tilde{\lambda}}\right) \notin T \mathbb{B}_{\tilde{\lambda}}$, i.e. $\left\|T u_{\tilde{\lambda}}(t)\right\|_{\infty}>\widetilde{\lambda}$ for some $x_{\tilde{\lambda}} \in J$ where $x_{\tilde{\lambda}}$ denotes $x$ depending on $\tilde{\lambda}$. But by using the previous hypotheses we have

$$
\begin{aligned}
& \left|u_{0}\right|+\|u\|_{\infty}\|a\|_{\infty} \frac{t^{\alpha}}{\Gamma(\alpha+1)}+\|g\|_{\infty} \frac{t^{\alpha}}{\Gamma(\alpha+1)}+\frac{K_{1}^{*} \mu_{1} x^{\alpha}}{\Gamma(\alpha+1)}+\frac{K_{2}^{*} \mu_{2} x^{\alpha}}{\Gamma(\alpha+1)} \\
& \leq\left(\left|u_{0}\right|+\frac{\|a\|_{\infty} \lambda+\|g\|_{\infty}+K_{1}^{*} \mu_{1}+K_{2}^{*} \mu_{2}}{\Gamma(\alpha+1)}\right)
\end{aligned}
$$

$$
\tilde{\lambda}<\left\|T u_{\tilde{\lambda}}\right\|_{\infty}
$$




$$
\begin{aligned}
& =\sup _{x \in J}\left|\left(T u_{\tilde{\lambda}}\right)(x)\right| \\
& \leq \sup _{x \in J}\left\{\begin{array}{c}
\left|u_{0}\right|+\left|\frac{1}{\Gamma(\alpha)} \int_{0}^{x}(x-s)^{\alpha-1} a(s)\right| u(s)|d s|+\left|\frac{1}{\Gamma(\alpha)} \int_{0}^{x}(x-s)^{\alpha-1} g(s) d s\right|+\frac{1}{\Gamma(\alpha)} \\
\int_{0}^{x}(x-s)^{\alpha-1}\left(\int_{0}^{s}\left|K_{1}(s, \tau)\right|\left|F_{1}(u(\tau))\right| d \tau+\int_{0}^{1}\left|K_{2}(s, \tau)\right|\left|F_{2}(u(\tau))\right| d \tau\right) d s
\end{array}\right\} d s \\
& \leq \sup _{x \in J}\left\{\left|u_{0}\right|+\|u\|_{\infty}\|a\|_{\infty} \frac{x^{\alpha}}{\Gamma(\alpha+1)}+\|g\|_{\infty} \frac{x^{\alpha}}{\Gamma(\alpha+1)}+\frac{K_{1}^{*} \mu_{1} x^{\alpha}}{\Gamma(\alpha+1)}+\frac{K_{2}^{*} \mu_{2} x^{\alpha}}{\Gamma(\alpha+1)}\right\} \\
& \leq \sup _{x \in J}\left(\left|u_{0}\right|+\frac{\|a\|_{\infty} \tilde{\lambda}+\|g\|_{\infty}+K_{1}^{*} \mu_{1}+K_{2}^{*} \mu_{2}}{\Gamma(\alpha+1)}\right) .
\end{aligned}
$$

Dividing both sides by $\widetilde{\lambda}$ and taking the limit as $\widetilde{\lambda} \rightarrow+\infty$, we obtain

$$
1<\frac{\|a\|_{\infty}}{\Gamma(\alpha+1)}
$$

which contradicts our assumption (29). Hence, for some positive integer $\tilde{\lambda}$, we must have $T \mathbb{B}_{\tilde{\lambda}} \subseteq \mathbb{B}_{\tilde{\lambda}}$.

An application of Schauder's fixed point theorem shows that there exists at least a fixed point $u$ of $T$ in $C(J, \mathbb{R})$. Then $u$ is the solution to (1) - (2) on $J$, and the proof is completed.

Theorem 5. If the series solution $u(x)=\sum_{m=0}^{\infty} u_{m}(x)$ obtained by the $m$-order deformation is convergent, then it converges to the exact solution of the fractional Volterra-Fredholm integrodifferential equation (1) - (2).

Proof. We assume $\sum_{m=0}^{\infty} u_{m}(x)$ converges to $u(x)$ then

$$
\lim _{m \rightarrow \infty} u_{m}(x)=0 \text {. }
$$

We can write,

$$
\begin{aligned}
\sum_{m=1}^{n}{ }^{c} D^{\alpha}\left[u_{m}(x)-\chi_{m} u_{m-1}(x)\right]= & { }^{c} D^{\alpha} u_{1}(x)+\left({ }^{c} D^{\alpha} u_{2}(x)-{ }^{c} D^{\alpha} u_{1}(x)\right) \\
& +\left({ }^{c} D^{\alpha} u_{3}(x)-{ }^{c} D^{\alpha} u_{2}(x)\right)+\cdots \\
& +\left({ }^{c} D^{\alpha} u_{n}(x)-{ }^{c} D^{\alpha} u_{n-1}(x)\right) \\
= & { }^{c} D^{\alpha} u_{n}(x) .
\end{aligned}
$$

Hence, from Eq.(33)

$$
\lim _{n \rightarrow \infty} u_{n}(x)=0 .
$$

So, using Eq.(34), we have

$$
\sum_{m=1}^{\infty}{ }^{c} D^{\alpha}\left[u_{m}(x)-\chi_{m} u_{m-1}(x)\right]=\sum_{m=1}^{\infty}\left[{ }^{c} D^{\alpha} u_{m}(x)-\chi_{m}{ }^{c} D^{\alpha} u_{m-1}(x)\right]=0 .
$$


Therefore from Eq.(34), we can obtain that

$$
\sum_{m=1}^{\infty}{ }^{c} D^{\alpha}\left[u_{m}(x)-\chi_{m} u_{m-1}(x)\right]=\hbar \sum_{m=1}^{\infty} \Re_{m-1}\left(\overrightarrow{u_{m-1}}(x)\right)=0 .
$$

Since $\hbar \neq 0$ and we have

$$
\sum_{m=1}^{\infty} \Re_{m-1}\left(\overrightarrow{u_{m-1}}(x)\right)=0 .
$$

By substituting $\Re_{m-1}\left(\overrightarrow{u_{m-1}}(x)\right)$ into the relation (35) and simplifying it, we have

$$
\begin{aligned}
\Re_{m-1}\left(\overrightarrow{u_{m-1}}(x)\right)= & \sum_{m=1}^{\infty}\left[{ }^{c} D^{\alpha} u_{m-1}(x)-a(x) u_{m-1}(x)-\int_{0}^{x} K_{1}(x, t) F_{1}\left(u_{m-1}(t)\right) d t\right. \\
& \left.-\int_{0}^{1} K_{2}(x, t) F_{2}\left(u_{m-1}(t)\right) d t-\left(1-\chi_{m}\right) g(x)\right], \\
= & { }^{c} D^{\alpha}\left(\sum_{m=1}^{\infty} u_{m-1}(x)\right)-a(x) \sum_{m=1}^{\infty} u_{m-1}(x) \\
& -\int_{0}^{x} K_{1}(x, t)\left[\sum_{m=1}^{\infty} F_{1}\left(u_{m-1}(t)\right)\right] d t \\
& -\int_{0}^{1} K_{2}(x, t)\left[\sum_{m=1}^{\infty} F_{2}\left(u_{m-1}(t)\right)\right] d t-\sum_{m=1}^{\infty}\left(1-\chi_{m}\right) g(x), \\
= & \left.{ }^{c} D^{\alpha} u(x)\right)-a(x) u(x)-\int_{0}^{x} K_{1}(x, t) F_{1}(u(t)) d t \\
& -\int_{0}^{1} K_{2}(x, t) F_{2}(u(t)) d t-g(x) .
\end{aligned}
$$

From Eq.(35) and Eq.(36), we have

$$
{ }^{c} D^{\alpha} u(x)=g(x)+a(x) u(x)+\int_{0}^{x} K_{1}(x, t) F_{1}(u(t)) d t+\int_{0}^{1} K_{2}(x, t) F_{2}(u(t)) d t,
$$

therefore, $u(x)$ must be the exact solution of Eq.(1) and the proof is complete.

\section{Illustrative example}

In this section, we present the analytical technique based on HAM to solve Caputo fractional Volterra-Fredholm integro-differential equation.

Example 1. Let us consider Caputo fractional Volterra-Fredholm integro-differential equation:

$$
{ }^{c} D^{0.75}[u(x)]=\frac{6 x^{2.25}}{\Gamma(3.25)}-\frac{x^{2} e^{x}}{5} u(x)+\int_{0}^{x} e^{x} t u(t) d t+\int_{0}^{1}\left(4-t^{-3}\right) u(t) d t,
$$

with the initial condition

$$
u(0)=0
$$


From (18), (37) can be written as

$$
\begin{aligned}
N[\phi(x ; q)]= & { }^{c} D^{0.75} \phi(x ; q)-\frac{6 x^{2.25}}{\Gamma(3.25)}+\frac{x^{2} e^{x}}{5} \phi(x ; q)-\int_{0}^{x} e^{x} t \phi(t ; q) d t \\
& -\int_{0}^{1}\left(4-t^{-3}\right) \phi(t ; q) d t .
\end{aligned}
$$

Now, using the $m^{t h}$-order deformation equation (24) and initial conditions (25), and recursive equation (27) we can write

$$
\begin{aligned}
u_{m}(x)= & \left(\chi_{m}+\hbar\right) u_{m-1}(x)+\hbar J^{0.75}\left[\frac{x^{2} e^{x}}{5} u_{m-1}(x)-\left(1-\chi_{m}\right) \frac{6 x^{2.25}}{\Gamma(3.25)}\right. \\
& \left.-\int_{0}^{x} e^{x} t u_{m-1}(t) d t-\int_{0}^{1}\left(4-t^{-3}\right) u_{m-1}(t) d t\right]
\end{aligned}
$$

Then,

$$
\begin{aligned}
u_{0}(x) & =0, \\
u_{1}(x) & =-\hbar x^{3}, \\
u_{2}(x) & =-\hbar(1+\hbar) x^{3}, \\
u_{3}(x) & =-\hbar(1+\hbar)^{2} x^{3}, \\
& \vdots \\
u_{n}(x) & =-\hbar(1+\hbar)^{n-1} x^{3},
\end{aligned}
$$

thus the HAM series solution can be written as

$$
u_{m}(x)=\sum_{n=0}^{m} u_{n}(x)=-\hbar\left[1+(1+\hbar)+(1+\hbar)^{2}+\cdots+(1+\hbar)^{m-1}\right] x^{3} .
$$

The approximate solution of (37) when $-2<\hbar<0$ is

$$
u(x)=\sum_{n=0}^{\infty} u_{n}(x)=-\hbar\left[1+(1+\hbar)+(1+\hbar)^{2}+\cdots\right] x^{3}=-\hbar\left(\frac{1}{1-(1+\hbar)}\right) x^{3}=x^{3} .
$$

\section{Conclusions}

Homotopy analysis technique has been successfully applied to derive approximate analytical solutions for Caputo fractional Volterra-Fredholm integro-differential equations. Also, we proved the existence and uniqueness of the solution. Moreover, the obtained results show that we can control of the convergence district of homotopy analysis technique by controlling the auxiliary parameter $\hbar$. The convergence theorem and the illustrative example establish the precision and efficiency of this technique. 


\section{References}

[1] M.AL-Smadi and G. Gumah, On the homotopy analysis method for fractional SEIR epidemic model, Research J. Appl. Sci. Engrg. Technol., 7 (18) (2014), 3809-3820.

[2] M. Bani Issa, A. Hamoud, K. Ghadle and Giniswamy, Hybrid method for solving nonlinear Volterra-Fredholm integro-differential equations, J. Math. Comput. Sci., 7 (4) (2017), 625-641.

[3] A. Hamoud and K. Ghadle, The reliable modified of Laplace Adomian decomposition method to solve nonlinear interval Volterra-Fredholm integral equations, Korean J. Math., 25 (3) (2017), 323-334.

[4] A. Hamoud and K. Ghadle, On the numerical solution of nonlinear Volterra-Fredholm integral equations by variational iteration method, Int. J. Adv. Sci. Tech. Research, 3 (2016), 45-51.

[5] A. Hamoud and K. Ghadle, The combined modified Laplace with Adomian decomposition method for solving the nonlinear Volterra-Fredholm integro-differential equations, J. Korean Soc. Ind. Appl. Math., 21 (1) (2017), $17-28$.

[6] A. Hamoud and K. Ghadle, Modified Adomian decomposition method for solving fuzzy Volterra-Fredholm integral equations, J. Indian Math. Soc., 85 (1-2) (2018), 1-17.

[7] A. Hamoud and K. Ghadle, Recent advances on reliable methods for solving Volterra-Fredholm integral and integro-differential equations, Asian Journal of Mathematics and Computer Research, 24 (3) (2018), $128-157$.

[8] A. Kilbas and S. Marzan, Nonlinear differential equations with the Caputo fractional derivative in the space of continuously differentiable functions, Differ. Uravn., 41 (1) (2005), 82-86.

[9] A. Kilbas, H. Srivastava and J. Trujillo, Theory and applications of fractional differential equations, NorthHolland Math. Stud. Elsevier, Amsterdam, 2042006.

[10] V. Lakshmikantham, Theory of fractional functional differential equations, Nonlinear Analysis: Theory, Methods and Appl. 69 (10) (2008), 3337-3343.

[11] S. Liao, The proposed homotopy analysis technique for the solution of nonlinear problems, Ph.D. thesis, Shanghai Jiao Tong University, 1992.

[12] S. Liao, Beyond Perturbation: Introduction to the Homotopy Analysis Method, Chapman and Hall/CRC Press, Boca Raton, 2003.

[13] X. Ma and C. Huang, Numerical solution of fractional integro-differential equations by a hybrid collocation method, Appl. Math. Comput., 219 (12) (2013), 6750-6760.

[14] K. Miller and B. Ross, An Introduction to the Fractional Calculus and Differential Equations, John Wiley, New York, 1993.

[15] R. Mittal and R. Nigam, Solution of fractional integro-differential equations by Adomian decomposition method, Int. J. Appl. Math. Mech., 4 (2) (2008), 87-94.

[16] S. Samko, A. Kilbas and O. Marichev, Fractional Integrals and Derivatives, Theory and Applications, Gordon and Breach, Yverdon, 1993.

[17] C. Yang and J. Hou, Numerical solution of integro differential equations of fractional order by Laplace decomposition method, Wseas Trans. Math., 12 (12) (2013), 1173-1183.

[18] X. Yang, Local fractional integral equations and their applications, Advs. Comput. Sci. Appl., 1 (4) (2012), 234-239.

[19] X. Zhang, B. Tang and Y. He, Homotopy analysis method for higher-order fractional integro-differential equations, Comput. Math. Appl., 62 (8) (2011), 3194-3203.

[20] Y. Zhou, Basic Theory of Fractional Differential Equations, Singapore: World Scientific, 62014.

[21] M. Zurigat, S. Momani and A. Alawneh, Homotopy analysis method for systems of fractional integrodifferential equations, Neur. Parallel Sci. Comput., 17 (2009), 169-186.

Department of Mathematics, Dr.Babasaheb Ambedkar Marathwada University, Aurangabad, 431004, India.

E-mail: drahmed985@yahoo.com; drahmedselwi985@gmail.com

Department of Mathematics, Dr.Babasaheb Ambedkar Marathwada University, Aurangabad, 431004, India.

E-mail: drkp.ghadle@gmail.com 